\title{
Nonlinear Concave Spiral Waves in Active Media Transferring Energy
}

\author{
Mikhail Mazurov,"* \\ ${ }^{1}$ Russian University Of Economics, RU-117997, Moscow, Russia
}

\begin{abstract}
Spiral concave autowaves are widely implemented in physics, chemistry, hydrodynamics, meteorology and other fields. A mathematical model of spiral concave autowaves based on the FitzhughNagumo equation and modified axiomatic models are presented. The existence of spiral concave autowaves transferring energy was predicted via computational experiments. Applications of spiral concave autowaves carrying energy in hydrodynamics, generation of tornadoes, breaking waves, and tsunamis and examples of such autowaves in biology and medicine are reviewed and the importance of concave spiral autowaves transferring energy is emphasized.
\end{abstract}

\section{Introduction}

Known autowaves in the active media of various types: straight, spiral, convex, concave, circular and others. It is established that concave spiral autowaves carrying energy are widely used in biology, medicine, hydrodynamics, physics, meteorology, cosmology. They are one of the essential mechanisms of self-organization. Autowaves in active media were discovered by B. p. Belousov [1]. There is a large number of works on the theoretical study of spiral convex autowaves and autowaves with rectilinear propagation fronts [2-5]. A number of applications of convex spiral autowaves are known.

Autowaves propagating rectilinearly and convex spiral autowaves (CSA) were first experimentally discovered in the Belousov-Jabotinsky reaction [1,2]. Spiral autowaves occur in nature everywhere in physical, chemical, biological, hydromechanical, geological systems. Spiral waves in a chemically active medium with topological charges equal to 1.4 are shown in Fig. 1. on the left.

CSA can be nested, the coefficient of attachment can be any. It is said that the topological charge of such an CSA is equal to the embedding coefficient. A distinctive feature of the CSA is its structure. The origin of the CSA occurs in the active medium from the unexcited nucleus. Around the core runs the tip of the spiral wave, generating a developing CSA. The nucleus of the CSA may be a hole (for example, the hole formed by the Vena cava in the atrium) around which the excitation wave circulates. In General, a circular nucleus is formed automatically during the evolution of the CSA. An important feature of this process is that the front of the autowave propagates through the active medium is not rectilinear, and twists in a spiral around the hole. The autowave touches the edge of the hole and rotates around the edge of the hole, becoming a source of circulating spiral waves in the active medium.

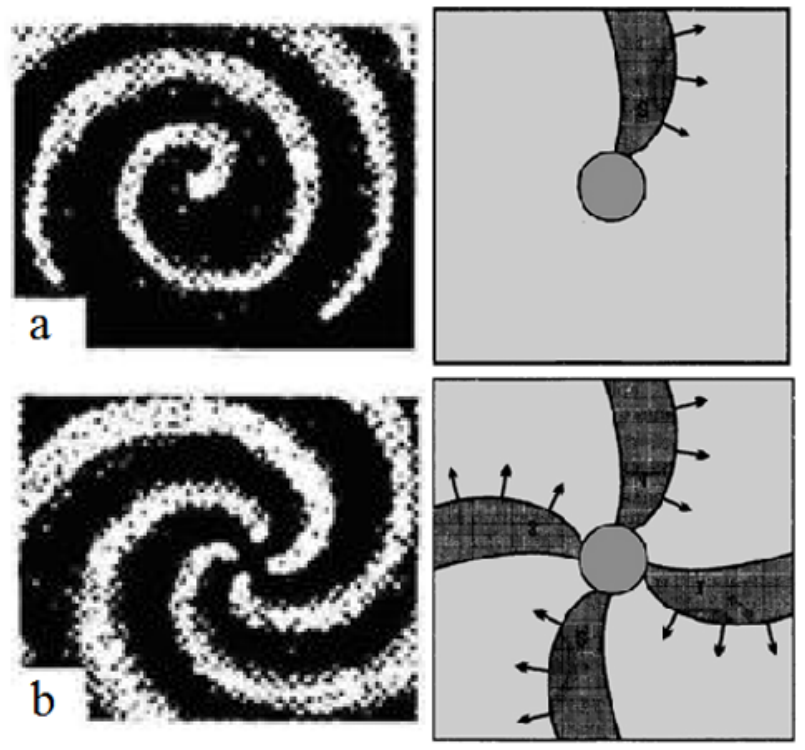

Fig. 1. Spiral waves in a chemically active medium with the values of topological charges equal to 1,4 , on the left. The schematic motion of the front (shown in black) of the spiral wave is shown on the right

Reverberators are called sources of CSA. in active media. The core of the reverb can be an anatomical hole, but it can also be a non-excitable zone, or, finally, a zone with significantly reduced excitability. The formation of such zones may be accompanied by cardiac pathology (necrosis, ischemia, etc.). The occurrence of spiral excitation waves around the holes of the hollow veins in the Atria explains the mechanism of a number of atrial arrhythmias. The structure of the formation of CSA. from the tip is illustrated in Fig. 1 on the left for cases where the topological charge is 1 and 4 . 
The properties of spiral waves are studied in detail in a number of theoretical and experimental works [2, 13]. In the circular circulation mode, the tip of the spiral wave rotates around a fixed area around a certain radius. Inside the circle bounded by this circle, the autowave does not penetrate. As we approach the center of rotation of the reverb, the amplitude of the excitation pulse decreases, and at a sufficiently low excitability of the medium in the center of the spiral wave there is a region of finite dimensions, where the amplitude of the excitation pulse is zero. This region of reduced amplitude at the center of the spiral wave is commonly referred to as the core of the spiral wave. The presence of such a region in the center of the spiral wave at first glance seems incomprehensible, since it always borders on excited areas. A detailed study of this phenomenon showed that the resting region in the center of the spiral wave retains its normal excitability, and the presence of a resting region in the center of the spiral wave is associated with the phenomenon of critical curvature of the autowave [13]. In the case of a homogeneous medium, the radius of the nucleus and the speed of rotation of the spiral are determined only by the properties of the medium itself, and not by the initial conditions. The size of the core of the spiral wave is due to the fact that the excitation wave circulating along the closed path must fit entirely on this path, without encountering its own refractory tail. The critical size of the spiral wave is understood as the minimum size of the medium in which the reverb can exist indefinitely in a homogeneous medium.

Recently, several new types of autowaves have been discovered, which have significant application value. A whole bunch of new types of autowaves is described in the works of V. K. Vanag [14-16]. These are, for example, perforated spiral autowaves, segment waves, accelerating waves, packet waves, etc. a Bouquet of autowaves of various types, including convex spiral autowaves, obtained and studied by V. K. Vanag, illustrated in Fig. 2

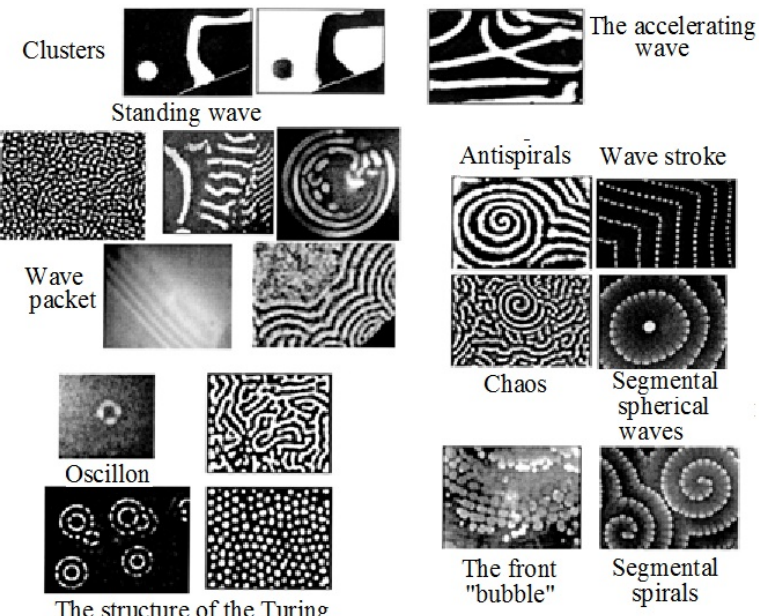

Fig. 2. Bouquet of autowaves of various types, including spiral autowaves, obtained and studied by V. K. Vanag [14-16]

\section{Concave nonlinear spiral autowaves}

\subsection{History of the discovery of concave spiral autowaves carrying energy}

Experimentally concave waves were first detected By V. K. Vanag and I. R. Epstein in chemical reactions with microemulsions in the form of aerosols [14-16]. In 2001, a fundamentally new type of spirals was discovered anti-spirals. If ordinary spiral waves always propagate from the core of the spiral outward, then in antispirals the waves move to the center. It is established that antispirals-anti-waves are phase waves, while ordinary spiral waves are usually impulse-trigger waves. Superficially antispirals little than differ from ordinary spirals. But if you look at their dynamics, the difference becomes obvious. The waves seem to self-originate at the boundary of the "watershed" between two basins of neighboring spirals and move to their centers, while in the case of ordinary spiral waves, the waves annihilate at the boundary of the "watershed" when they collide. Very often spiral and concentric waves are observed simultaneously. This applies to both normal waves and anti-waves.

It was further established that the anti-spiral and anticoncentric waves are due to wave instability. The behavior of antispiral waves is typical for phase waves and impossible for trigger waves. Therefore, waves in antispirals are phase waves that occur in an oscillating medium. As writes W. K. Vanag, in antispiral waves he saw the, what not can be. Understanding of what he saw came later and was associated with negative dispersion in the presence of wave instability [14-16].

\subsection{Theoretical studies of phase concave autowaves on the Fitzhugh-Nagumo mathematical model}

The first works on the theoretical study of phase concave waves and their implementation in a computational experiment belong to O. A. Mornev, M. I. Tsyganov, Aslanidi, M. A. Tsyganov [17-19].

O. A. Mornev et al. in numerical experiments with a system of reaction-diffusion Fitzhugh - Nagumo equations describing two-dimensional self-oscillating active media, solutions corresponding to a phase concave spiral wave rotating stationary around a circular obstacle in a medium of finite dimensions are found and investigated. The calculations used a system of equations of the Fitzhugh - Nagumo type, describing a two-dimensional self-oscillating active medium

$$
\begin{aligned}
& \frac{1}{R}\left(\frac{\partial^{2} V}{\partial x^{2}}+\frac{\partial^{2} V}{\partial y^{2}}\right)=C \frac{\partial V}{\partial t}+I_{F}(V)+I, \\
& \frac{\partial I}{\partial t}=\frac{I_{S}(V)-I}{\tau(V)},
\end{aligned}
$$

$I_{F}(V) \equiv k V\left(V-V_{t h}\right) \cdot\left(V-V_{A}\right), \quad I_{S}(V) \equiv \sigma V$, 


$$
\tau=\left\{\varepsilon_{0}+\Delta \varepsilon\left(2-t h \frac{V-V_{1}}{u_{1}}+t h \frac{V-V_{2}}{u_{2}}\right)\right\}^{-1},
$$

Where ${ }^{x}$ and ${ }^{y}$ are spatial coordinates; the meaning of other variables and parameters is deciphered in [17, 18]. The parameter $\Delta \varepsilon_{\text {was set to }} \Delta \varepsilon=0.60$; the values of the other parameters were the same as in [17, 18]. With this setting of parameters in a twodimensional excitable medium, the mode of formation of a concave spiral autowave is realized. Such a wave is realized in the field of mode parameters in the form of a doublet structure. A doublet structure is a structure when the main excitation pulse in the active medium is followed by a second excitation pulse of much smaller amplitude, which allows to exceed the threshold of relative refractoriness. The active medium thus becomes self-oscillating. That allows you to appear to a phase autowave. The emerging waves are phase of the concave waves, which do not carry energy.

\subsection{Properties of phase autowaves}

To elucidate the properties of phase waves in oscillating active media, we consider phase autowaves in onedimensional and two-dimensional media. Let the set of one-dimensional phase wave with positive and negative dispersion, respectively

$$
u=A \cdot \sin (\omega(x+k t)) ; u=A \cdot \sin (\omega(x-k t))
$$

These equations describe a sine wave, the first of

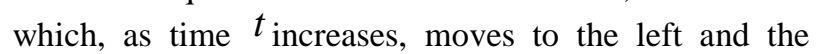
second to the right. According to calculations the energy flow $W$ through any cross section area $\mathrm{S}$ is equal to

$$
W=\int_{0}^{2 \pi / \omega} A^{2} S \sin ^{2}(\omega(t \pm k x)) d t=\frac{\pi}{\omega} A^{2} S
$$

It follows that phase waves do not transfer energy, and the possibilities of their application are limited. Consider two-dimensional spiral concave waves on the example of waves described by the equations

$$
r=a \cdot(\varphi+k t) ; \quad r=a \cdot(\varphi-k t) .
$$

These equations describe a spiral wave, the first of which is concave and the second is convex. It can be shown that phase waves do not transfer energy in the two-dimensional case.

Concave of the phase waves do not carry energy. There was a fundamental question: whether there are concave spiral autowaves transferring energy. Was tasked with modeling concave spiral waves carrying energy (CSACE). Concave phase-type autowaves do not transfer energy. Therefore, the task of creating concave autowaves of trigger type, transferring energy, was set. A system of two nonlinear partial differential equations is used to study nonlinear spiral waves.

\subsection{Concave spiral waves that carry energy}

Let us consider theoretically the production of concave spiral autowaves carrying energy using the simulation of autowave processes in active media. All the above cases of concave formation show that it is possible to obtain only phase concave spiral autowaves that do not transfer energy in self-oscillating active media. The formation of CSACE that transports energy, in a homogeneous twodimensional region is impossible. It has been suggested that the CSACE can be created in an inhomogeneous region in the presence of areas with a decreasing speed of propagation of the autowave. This assumption was confirmed. For a concrete implementation of CSACE was used inhomogeneous circular region divided into the ring. A system of two nonlinear partial differential equations, namely the Fitzhugh-Nagumo equation, was used as the active medium for the generation of CSACE

$$
\begin{aligned}
& \frac{\partial u}{\partial t}=C \varepsilon^{-1}\left(u-\frac{u^{3}}{3}-v\right)+D \Delta u, \\
& \frac{\partial v}{\partial t}=\varepsilon(u+\beta-\gamma v),
\end{aligned}
$$

where: $\Delta u$ - Laplace operator; $\varepsilon=0.03, \beta=0.7$, $\gamma=0.8$. parameters . These equations are known to be the most suitable for the conceptual study of spiral waves.

The parameters of the equation in each ring were chosen in such a way as to provide the necessary velocity of propagation in each ring for the formation of a concave autowave. This speed should decrease as the outer and inner diameters of the ring decrease. In the center of the ring, a small diameter non-excitable region is created, since as the diameter of the non-excitable region decreases, a decrease in the propagation velocity is necessary, so that when the diameter tends to zero, the velocity must also decrease to zero, i.e. for example $d \rightarrow 0 \Rightarrow v \rightarrow 0$, it is impossible to realize without the presence of a non-excitable region of small size, such as a circle of small diameter. Thus, the autowave velocities in the rings should decrease as they approach the center, for example for a region of five rings: $V_{5}<V_{4}<V_{3}<V_{2}<V_{1}$. When using the FitzhughNagumo equations, the decrease in the velocity of the autowave propagation was achieved by reducing the diffusion coefficient $D$, since as is known from the theory, the velocity of the autowave propagation is proportional $\sqrt{D}$

Consider the results of a computational experiment to create a concave wave in a ring-shaped inhomogeneous active medium using the Fitzhugh-Nagumo equations. The speed of propagation of the autowave decreases from the periphery to the center. The concave autowave is shown in Fig. 3. on the left. 


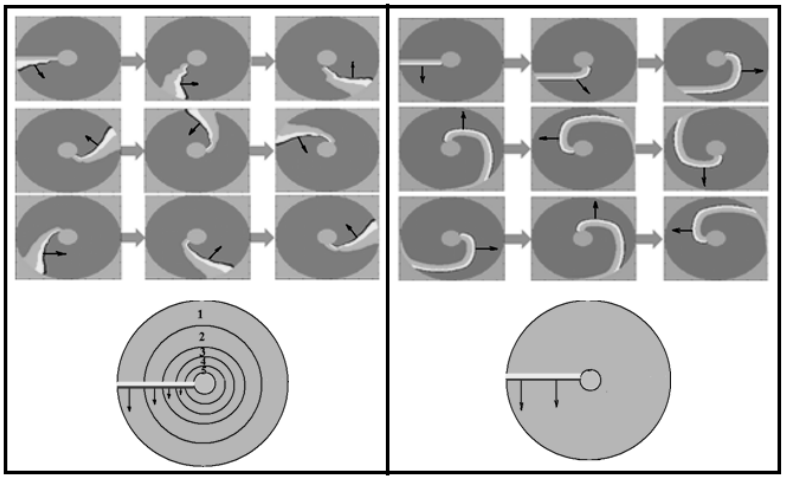

Fig. 3. Initial data for the formation of a concave autowave (left) in the Fitzhugh - Nagumo model. The velocity of the autowave propagation in the selected bands decreases from the periphery to the center due to an increase in the diffusion coefficient. The direction of wave propagation is shown by arrows. The initial data for the formation of a conventional spiral autowave (right) in the Fitzhugh - Nagumo model, the wave propagation region is uniform, the velocity of the autowave propagation at each point is the same.

When modeling a concave autowave, a serious problem is to obtain ring regions with decreasing autowave propagation velocities to the center. The possibilities of modeling different propagation velocities using the Fitzhugh-Nagumo equation are limited. Therefore, to implement effective modeling, we will use the axiomatic model [6]. Let's give a description of this model.

\subsection{Axiomatic model for the description of electrical processes in the membrane}

An effective axiomatic model for the study of autowave processes in distributed active media was proposed in [6, 22]. The model was used to study the interaction in the system of many globally related elements of the environment. Model description includes [6, 22]: 1). The numerical assignment of shapes of the excitation pulse in excess of a dynamic threshold of excitation; 2) Numerical dynamic threshold task (forms threshold relative refractoriness); and 3). Excitation of the active medium element occurs when the dynamic threshold is exceeded; 4). The state of all elements is characterized by a state matrix. The number of the matrix element characterizes the moment of time of the excitation pulse of the medium element and the dynamic threshold. We present the results of computational modeling of concave autowave in the axiomatic model. The modeling area is a circular area divided into 7 layers-rings. An axiomatic model with a connection to the nearest neighbor is used. The highest rate of propagation of the autowave is realized in the first layer, in other layers it decreases. At the initial moment of time, the excitation is set on the line from the edge to the center of the circle. The results of computational modeling of the concave wave are illustrated in Fig. 4.

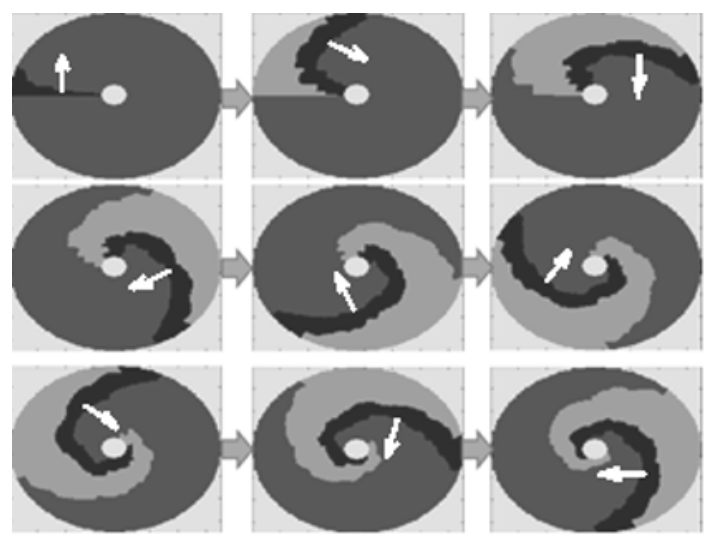

Fig. 4. Concave autowave in axiomatic model. The color coloring in the output is as follows: black-a point in the state of excitation, light gray-a point in the state of relative refractoriness, dark gray-a point at rest. The total delay from the head to the tail-about $3 / 4$ of the treatment period.

From the physical point of view, the excitation of a concave autowave transferring energy in the presence of nested ring regions with a decreasing excitation rate with a decrease in the diameter of the rings can be considered as a sequential synchronization of autowaves in these ring regions. Only studies of the interaction of autowaves for the case of their rectilinear propagation in nerve fibers are known in the works of Katz, Schmitt, V. S. Markin and others [20].

\section{Areas of existence and application of concave spiral autowaves transferring energy}

\subsection{General characteristics of applications}

The study of autowave processes showed that concave spiral autowaves carrying energy CSACE are widely represented in various natural processes and have a significant number of applications. The number of such applications is very large, and the application takes place in a variety of areas. It is established that concave autowaves carrying energy are widely used in physics, hydrodynamics, biology, meteorology, cosmology. They are one of the essential mechanisms of self-organization.

\subsection{Concave autowaves in hydrodynamics}

Unlike phase concave waves in the self-oscillating active medium, trigger concave spiral autowaves CSACE are energy carriers from the periphery to the center. This allows a significant number of concave wave applications to be implemented. Let us consider the realizations CSACE when bodies flow around a liquid [19-25]. 

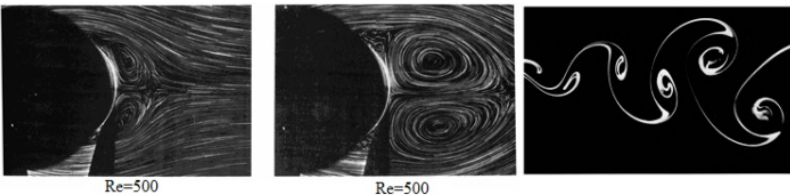

Fig. 5. On the left CSACE when flowing around a circular cylinder, on the right track of Karmein.

\subsection{Concave autowaves in the formation of atmospheric tornadoes}

The mathematical model of tornado formation is presented in [19-25]. The storm cloud in which a tornado forms is a vapor-air mixture saturated with energy. The source of energy is a vapor substance, the condensation of which to a liquid droplet state is associated with a significant release of energy. Thus, a thundercloud is an active environment in CSACE occur. In this case, the clouds formed CSACE lead to a twist of the air medium lying inside CSACE, and the formation of a vortex tornado. Tornadoes can form in other environments. Distinguish atmospheric tornadoes. fire, dust, air, snow and other [19-25]. The different types of tornadoes are illustrated in Fig. 6.

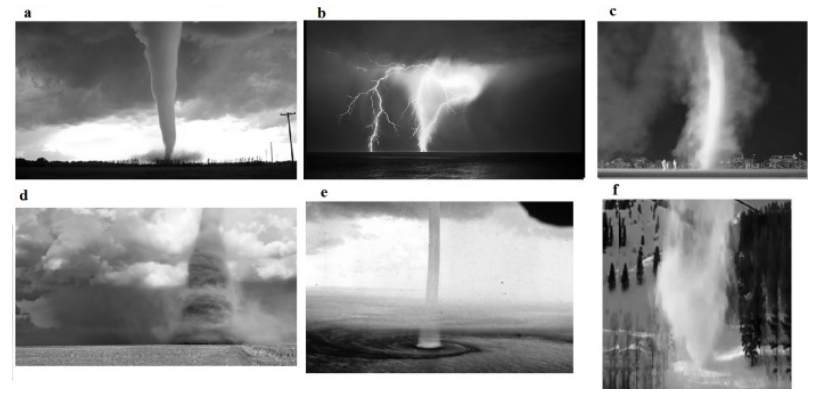

Fig. 6. CSACE generators the formation of a tornado. The figure shows: a - atmospheric tornado; b-lightning tornado; c fire tornado; d-dusty "devil"; e-water tornado; f-snow tornado.

BCAAs are the main mechanism of cyclone formation. The difference between cyclones and tornadoes is that tornadoes are realized in storm clouds at a distance of about a kilometer, and cyclones are realized in areas of about a dozen and hundreds of kilometers. The General property of these grandiose natural phenomena is the General mechanism of formation on the basis of CSACE. A photograph of the cyclone, representing a Grand concave wave, is shown from space in Fig. 7.
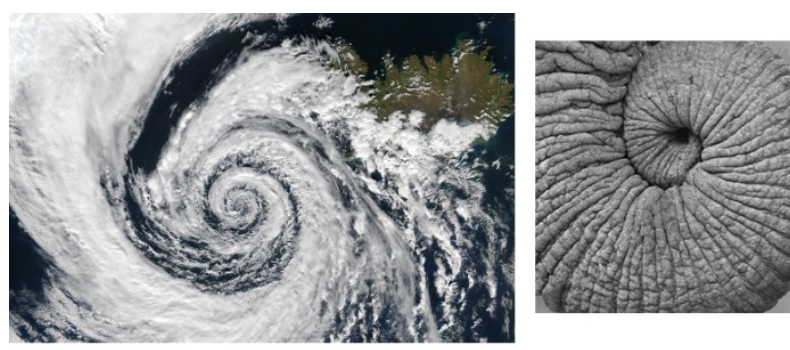

Fig. 7. Photo of the cyclone from space. Pay attention to the similarity of the shape of the cyclone with the shape of the folded trunk of the elephant, shown on the right.

\subsection{Concave autowaves in the formation of wind and surf waves}

The formation of surf waves in the presence of wind blowing parallel to the water surface is shown in Fig. 8 on the left. For fig.. 8 right shows a photograph of the coastal waves, which are essentially CSACE [19-25]

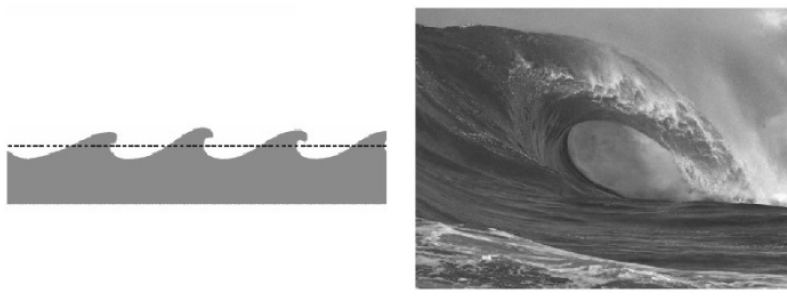

Fig. 8. The formation of surf waves in the presence of wind blowing parallel to the surface of the water on the left, the right shows a photo of the coastal wave, which is in fact the CSACE

\subsection{Concave waves in tsunami formation}

A concave wave is a tsunami. Tsunami - long waves generated by a powerful impact on the entire water column in the ocean or other body of water. The causes of tsunamis: earthquakes, volcanic eruptions and other underwater explosions (including explosions of underwater nuclear devices), landslides, glaciers, meteorites and other destruction above or below the water level-all have sufficient potential to cause a tsunami [19-25].

In the formation of a tsunami, a significant role is played by the decrease in the depth of the sea as it approaches the shore. The lower part of the resulting wave is inhibited, its speed decreases. The upper part of the wave has a greater velocity, which leads to the formation of USAPA. The scheme of tsunami formation is shown in Fig. 9 on the left, the destructive power of the tsunami, illustrated by a painting by a Japanese artist, is shown in Fig. 9 to the right.
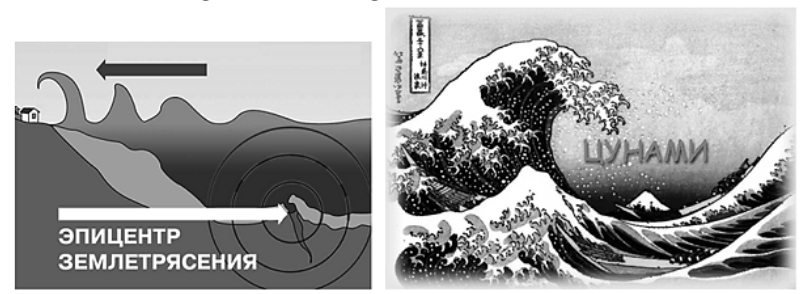

Fig. 9. The scheme of tsunami formation is on the left. Picture of a Japanese artist with an illustration of the tsunami-on the right.

\subsection{Concave autowaves in biology}

Let's consider examples of realization of concave autowaves in biology. Several examples have been collected where concave autowaves are effectively used by animals, humans, plants in various cases of adaptation to the environment.

Many plants and animals have different organs for grasping (antennae of plants, trunk of an elephant, 
prehensile tails of climbing animals). All of them have a rough surface to increase the friction force [19-25].

Among living organisms are common devices (wool, bristles, scales, spikes, located obliquely to the surface), thanks to which the friction is small when moving in one direction and large-when moving in the opposite direction. Adhesion is achieved either by sharp points on the limbs, or by small irregularities, such as bristles, scales, tubercles. Considerable friction is also necessary for the grasping organs. Their form is interesting: it is either forceps grasping an object from two parties, or the strands encircling it. The hand combines the action of forceps and full coverage on all sides; the soft skin of the palm is well connected with the roughness of objects that need to be held.

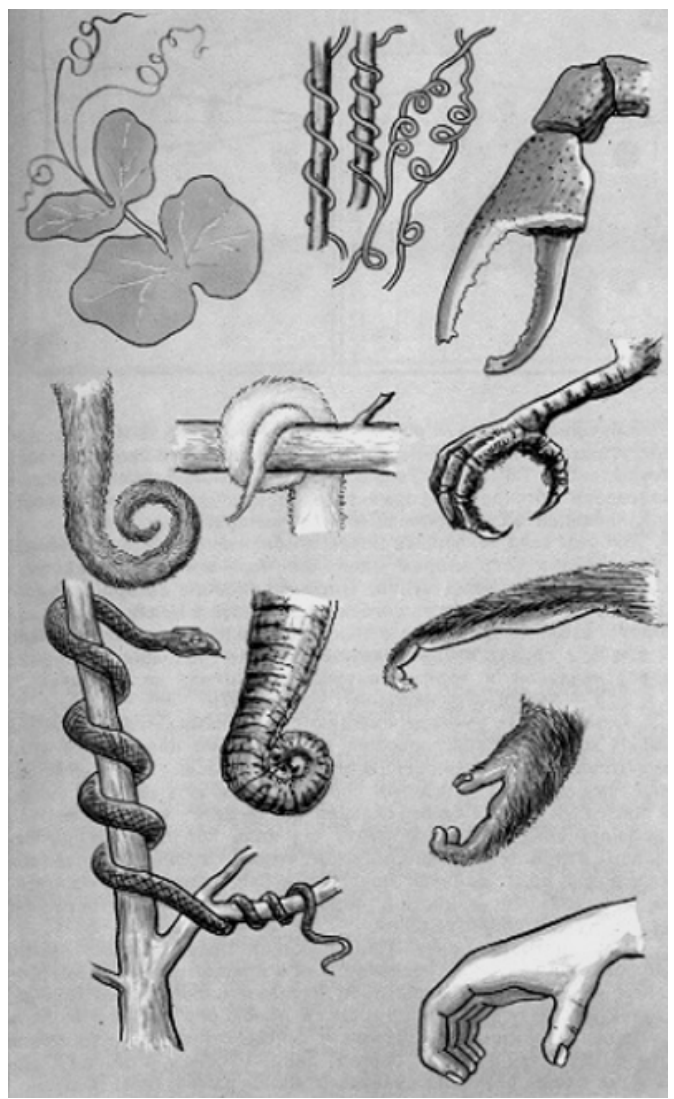

Fig. 10. Use of concave autowaves in biology.

For fig. 10 presents: top left and center are shown concave waves, servants winding plants - morning glories, vines to consolidate and move up, right claw of crustaceans, having the concavity toward the center; in the second row left and center are shown concave waves in the form of prehensile tails tree-climbing animals like monkeys, tree-climbing marsupials, the tails are used for fixing on branches up and down; in the second row on the right shows the concave autowalla in the form of a clawed bird's feet; the bottom row is concave avtovolna implemented in the body of a boa constrictor and other snakes in the form of elephant trunk; in the lower row on the right - the upper limbs of man and Primate in the form of a concave autowave.
The forward and reverse twisting of the elephant's trunk is illustrated in fig. 11.
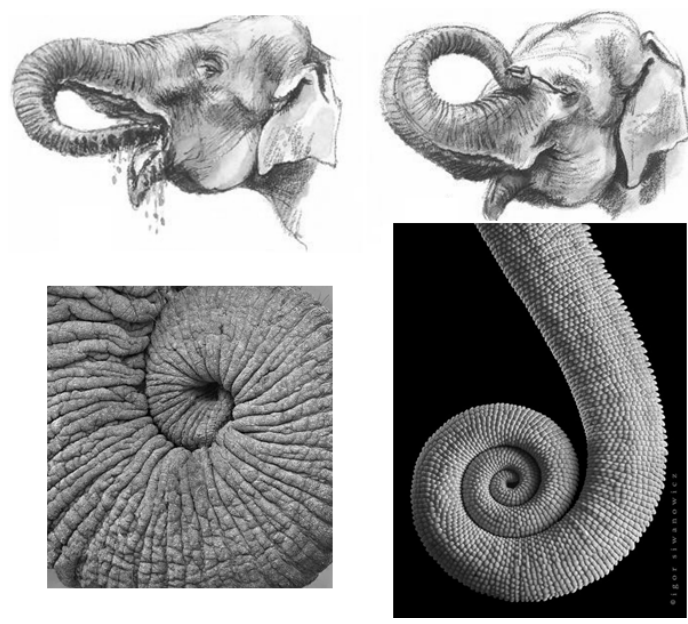

Fig. 11. Forward and reverse twisting of the elephant trunk, drawings from the top left and right and bottom left. Twisting the tail of the reptile-figure bottom right.

The peculiarity of concave spiral waves is clearly visible - the reduction of the transverse size as it approaches the center.

The use of concave wave properties can be illustrated by flexing the fingers of the hand. An illustration of the three positions of the fingers of the hand when bending is shown in fig. 12.
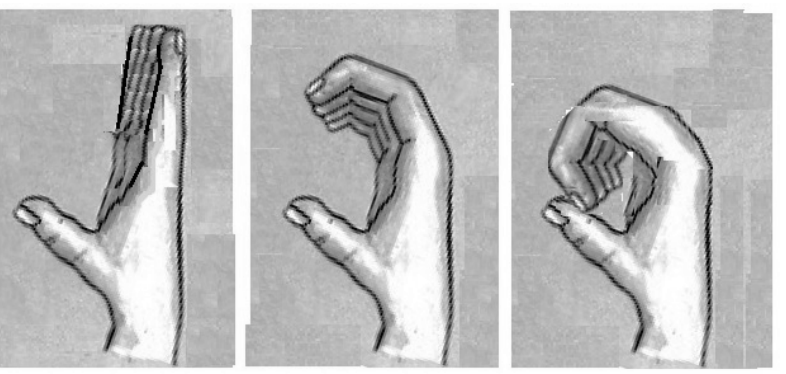

Fig. 12. Illustration of the three positions of the fingers of the hand.

The realization of the structure characteristic of the concave spiral autowave is achieved by successive flexion of the hand and parts of the fingers from the thicker part to the thinnest-the fingertips.

\subsection{Concave autowaves carrying energy to the heart}

Let us consider the muscular structure of the heart, realizing the mode of blood movement, based on the use of the properties of concave spiral autowaves, transferring energy to realize the vortex tornado-like blood movement. The muscle fibers of the heart spiral down to the top of the heart, and then rise again to its base, as shown in fig. 13, and [26, pp. 89-91]. 

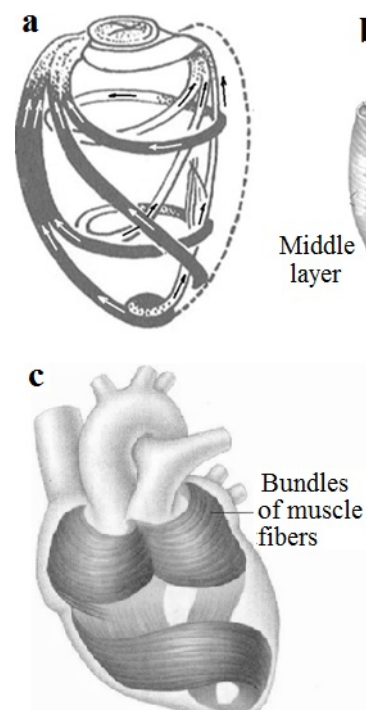

d

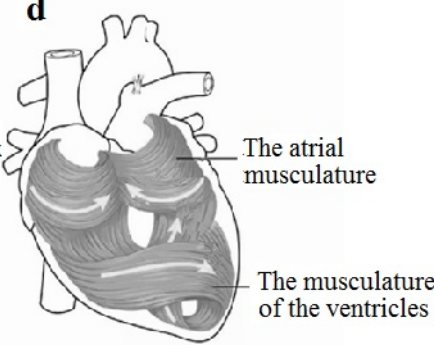

Fig. 13. Schematic representation of the structure of muscle fibers of the heart [26, p. 89-91]. Each bundle of fibers encircles the ventricles of the heart, from the top of the heart to the aorta a) Muscle fibers of the heart spiral down to the top of the heart, and then rise again to its base, forming a chiral system [26, pp. 89-91]. b) shows the anatomical structure of the muscle fibers of the heart. Each bundle of fibers encircles the ventricles of the heart from the top to the base, forming a chiral fiber system. b) shows the anatomical structure of the muscle fibers of the heart. The muscle fibers of the atrium also form the chiral system, as seen in Fig. 13 in c,d..

The anatomical structure of the heart when the epicardium is removed, illustrating the muscular layer of the ventricles of the heart, when viewed from the apex of the heart, is shown in Fig. 13, b. in Fig. 13, b shows the muscle layers of the heart: outer layer, middle layer, deep layer. The contractions of all three layers add up, increasing the efficiency of blood ejection. Pumping of the heart with blood after its ejection into the aorta, pulmonary and coronary is carried out with the help of synchronous injection of blood from the Atria. It should be noted that the presence of three duplicate muscle layers of the heart increases its stability in the event of pathological changes, such as myocardial infarction or other damage to the structures of the heart. The mechanical work of the Atria is also carried out by the realization of concave spiral autowaves, transferring energy and realized by the muscular structure of the Atria, as shown in Fig. 13, c, d. The movement of blood in the ventricles of the heart is similar to the suction center of the whirlpool, to which the blood rushes from the periphery with increasing speed, to return back at a slower pace and disperse throughout the body [26, pp. 89-91]. The muscle fibers of the heart form a concave wave of mechanical contraction in the absence of a rigid skeleton in the heart (there are only flexible fibrous fibers in the heart). The internal endocardial structure of the heart can be compared to a flexible rough , tightened by the chiral structure of muscle fibers during systole and inflated during diastole during blood filling. It is interesting to note that at the end of the systole there is a circular motion similar to the wringing of linen by a laundress, which allows for a more complete expulsion of blood from the ventricles of the heart. There are three layers of muscle fibers, each of which is made of concave spiral autowaves, carrying energy, providing the ejection of blood from the valves located near the Atria.

Understanding the mechanism of contraction of the muscles of the heart, which occurs under the influence of external Electromechanical excitation, may be useful in the treatment of myocardial infarction. When myocardial infarction occurs partial damage to the muscles of the heart due to partial hemorrhages. Depending on its localization of damage, it is possible to predict the treatment plan and elimination of the consequences of myocardial infarction. Note that the study of macroscopic mechanical activity of the heart requires further experimental and practical studies.

\subsection{The mechanisms of tornado formation in the heart}

The main macroscopic function of the heart is the effective release of blood into the aorta of the thoracic and pulmonary. In a number of works, it was convincingly shown that an important feature of the flow in the ascending aorta and the aortic arch in most healthy people is the swirling of the blood flow clockwise along the bloodstream [27-30]. To solve these problems, evolution used the following solutions: 1) tornado-like swirling of the blood flow (such as a tornado); 2) the special structure of the ventricles of the heart of the right and left in the form of a muscle structure, providing a swirl of the blood flow and the inner surface of the endocardium in the form of a structured rough wall to ensure an increase in blood flow in accordance with the twisting movements of the walls.

In fact, the heart is resilient sacs, the left and right ventricles, filled with liquid blood and having relatively small openings for ejection of blood, chest and pulmonary aorta. The quick and effective release of a viscous blood fluid through small openings is a rather difficult task. This can be easily verified by pushing water out of a rubber enema by pressing on a wide part of it.

\subsection{The sequence of the formation of tornado- like blood flow in the heart}

To ensure an uninterrupted and unstressed flow, at each collision of the incoming and residual flows, they must be "stitched", accompanied by the exchange of circulation and mass. Since we are talking about swirling flows, the condition for continuous "stitching" is the unidirectional swirling of both flows, and then the flows join "beginning to end". It is impossible to restore the conditions of "cross-linking" the flows by analyzing the spatial orientation of the trabeculae in the mold, which is the imprint of the instantaneous state of the flow. Nevertheless, such an analysis makes it possible to determine the nature of the effects exerted by the trabeculae on the swirling blood flow at a certain point in the cycle and to present on this basis. how the flow came to this state and will evolve subsequently. Thus, the 
analysis allows us to restore the picture of the evolution of intracardiac blood flow, based on the hydrodynamic parameters calculated from the casts of the cavity of the left ventricle of the heart [27-30].. The stages of evolution of the intracardiac blood flow are schematically shown in fig. 14.
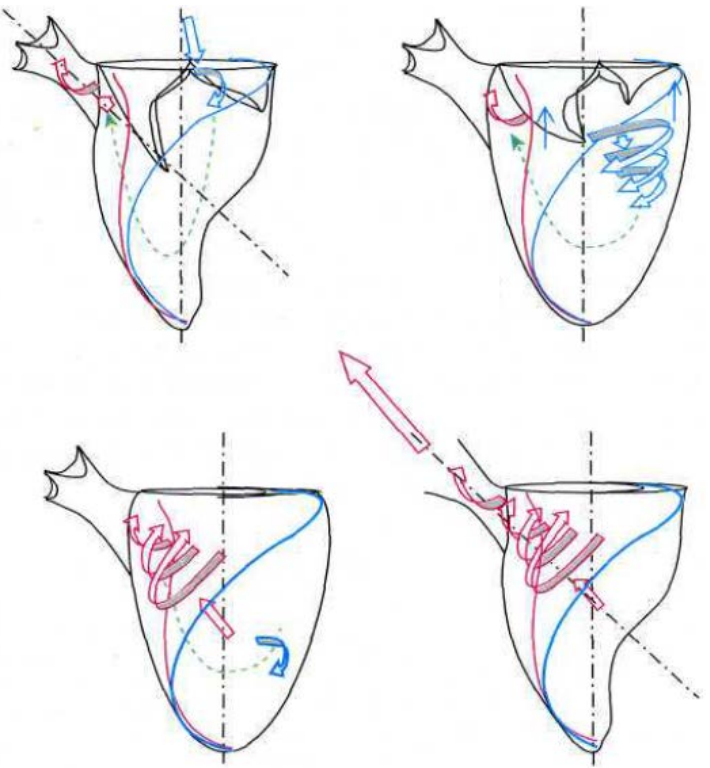

Fig. 14. Scheme of the main stages of the evolution of blood flow inside the cavity of the left ventricle.

The wide blue arrows indicate the inflowing stream, the red arrows indicate the expelled stream, the thin blue arrows indicate the return flows, the green arrow indicates the direction of crosslinking of the inflowing stream and the stream formed by the residual volume; the blue line is the trabecula of the diastolic relief of the cavity, the red line is the trabecula of the systolic relief.

To verify the theoretical premises explaining the tornado-like movement of blood, experiments were performed with visualization of the motion of a model fluid using particles. The result of the experiments is illustrated in fig. 15.
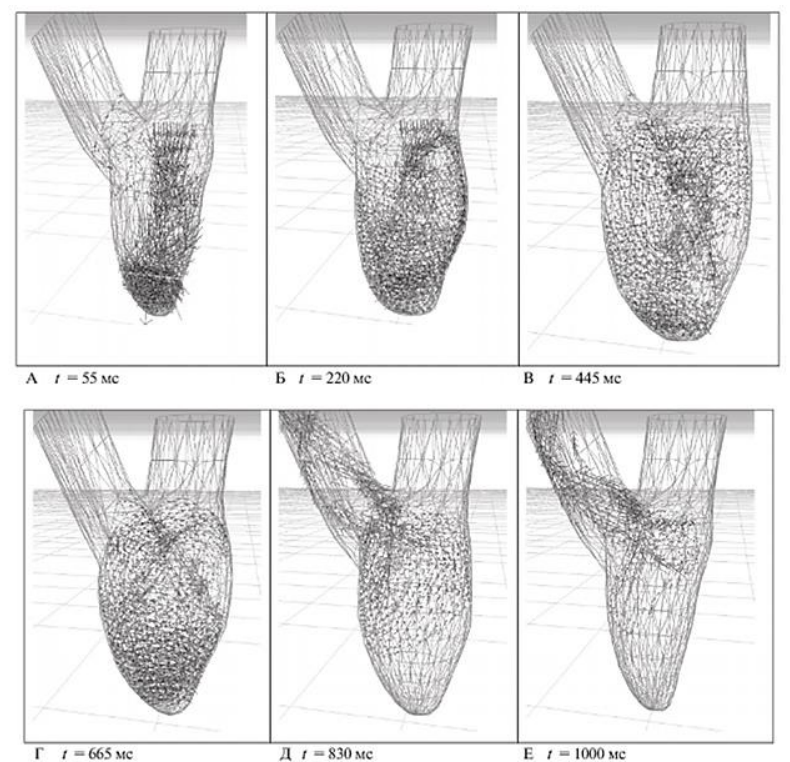

Fig. 15. Visualization of the flow of a model fluid with simulated exposure to C5 trabeculae. The particle velocity is shown in shades of gray. Black color corresponds to the maximum particle velocity, conventionally taken as $1 \mathrm{~m} / \mathrm{s}$, white - to the minimum zero velocity.

\section{Conclusion}

The existence of spiral concave autowaves transferring energy in the active medium is predicted. For the first time, spiral concave autowaves carrying energy were discovered by V.K. Vanagom and I.R. Epstein in experimental studies in 2001. Then, phase concave spiral autowaves in self-oscillating active media were studied in a computational experiment by O.A. Morynev et al. In this work, the existence of concave spiral autowaves transferring energy was predicted. The existence of such CSACEs is possible in heterogeneous active media of a certain structure. It has been established that spiral concave autowaves carrying energy are widely represented in various natural processes and have a significant number of applications. It has been established that concave autowaves carrying energy are widely used in physics, hydrodynamics, biology, meteorology, cosmology. This paper describes the applications of CSACE in biology and medicine. CSACE s, as well as well-known convex spiral autowaves, are one of the essential tools for selforganization.

\section{References}

1. B.P. Belousov on Sat Autowave processes in systems with diffusion. (Gorky: Publishing House of the GGU, 1951)

2. A.M. Jabotinsky Concentration fluctuations. (Nauka, Moscow, 1974.)

3. R.J. Field., R.M. Noyes. J. Chem. Phys. 60, 1877 84. (1974).

4. A.T. Winfree, Science 175, 634 (1972)

5. A.T. Winfree, Review 32(1), (1990)

6. M.E. Mazurov "Nonlinear synchronization and rhythmogenesis in the electroexcited heart systems" Diss. ... d.f.-m. n (Pushchino 2007)

7. M.E. Mazurov Biophysics 54(1), 81-88 (2009)

8. M.E. Mazurov, Biophysics 54(1), 89-96 (2009)

9. M.E. Mazurov, Reports of the Academy of Sciences. 444(1), 31-34 (2012)

10. A.V. Ardashev, M.E. Mazurov, I.M. Kalyuzhny, E.G. Zhelyakov,., Yu.N. Belenkov. Cardiology, 7, 50-55 (2012)

11. A.V. Ardashev, E.G. Zhelyakov, I.M. Kalyuzhny, V.A. Finko, Yu.N. Belenkov Cardiology, 4, 39-45 (2014)

12. M.E. Mazurov, I.M. Kalyuzhny. Bulletin of Moscow State University. Series: Physics, Astronomy, 3, 45-49 (2014)

13. V.S. Zykov Modeling wave processes in excitable media. (Science Moscow 1968)

14. V.K. Vanag, Phys. 174(9), 991 (2004). 
15. V.K. Vanag, Dissipative Structures in ReactionDiffusion Systems: An Experiment and Theory (RHD 2008).

16. V.K. Vanag, I.R. Epstein ,. Science. 294, (5543) 835 (2001)

17. O.V. Aslanidi, O.A. Mornev Mat. modeling. 11(9), 3-22 (1999)

18. O.A. Mornev, O.V. Aslanidi, L.M. Chaylakhyan. DAN. 353, 682-686 (1997).

19. M.E. Mazurov, I.M. Kalyuzhny. In: Collection of reports of the $\mathrm{V}$ International Conference "Mathematical Biology and Bioinformatics", ed. In D. Lakhno (MAKS Press. Moscow, 49-50 2014)

20. V.S. Markin, V.F. Pastushenko, Yu.A. Chizmadzhev, Theory of excitable media. (Science Moscow 1981).

21. M.E. Mazurov Izv. RAS Physical Series 82(1), 7277 (2018)

22. M.E. Mazurov, Mathematical Biology and Bioinformatics, 13(1), 187-207 (2018). doi: 10.17537 / 2018.13.187

23. M.E. Mazurov, Izv. RAS. Physical Series, 83(1), 111-117 (2019)

24. M.E. Mazurov, Earth and the Universe, 2, 56-68 (2019)

25. M.E. Mazurov, Identification of mathematical models of nonlinear dynamical systems. Monograph. (Lenand. Moscow 2019)

26. T. Schwenk Feeling chaos. The formation of moving forms in water and air. (New Center Moscow 2003)

27. G.I. Kiknadze, Yu.K. Krasnov. DAN. 290(6), 13151319 (1986)

28. G.I. Kiknadze, V.G. Oleinikov, I.A. Gachechiladze et al. DAN. 351(1), 119-122 (1996).

29. JI.A. Boqueria, G.I. Kiknadze, I.A. Gachechiladze., R.F. Gabidullina, V.N. Makarenko, A.Yu. Gorodkov, Clinical physiology of blood circulation. 4, 18-28 (2011).

30. A.Yu. Gorodkov, Quantitative analysis of the structural organization of the pulsating blood flow in the left ventricle of the heart and aorta. Dis. Dr. Biol. sciences. 03.00.13 (Moscow 2004) 